\title{
On exceptions to Szegedy's theorem
}

M. Chammovich, G. Freiman

and I. SCHONHEM (Tel-Aviv)

Thanks once more to Professor Erdös for so many things he did for us

1. Introduction and main result. Finally the attempts $[1],[3],[5],[6]$, [7] to answer a question of R. L. Graham on

$$
\max _{\substack{i \neq j \\ i \leqslant n \\ j \leqslant n}} \frac{a_{i}}{\left(a_{i}, a_{j}\right)}
$$

ended in the following result of $\mathrm{M}$. Szegedy:

If $n \geqslant n_{0}$, where $n_{0}$ is an explicitly computable constant, then for any $n$ distinct positive integers $a_{1}, a_{2}, \ldots, a_{n}$ :

$$
\max _{i, j} \frac{a_{i}}{\left(a_{i}, a_{j}\right)} \geqslant n
$$

This beautiful result being asymptotic; the way is not closed for combinatorially minded reseatrchers to look for possible exceptions or to look for a proof that no exceptions exist.

For some particular eases this has been done in the papers cited above.

The main result of this paper is formulated in the following theorem:

THEOREM 1. Let $a_{1}<a_{2}<\ldots<a_{n}$ be natural integers, $n \geqslant 2$. If $s$ is the smallest number of primes such that each $a_{i}, i=1,2, \ldots, n$ is a product of powers of those primes and $s \leqslant 5$ then

$$
\max _{i, j} \frac{a_{i}}{\left(a_{i}, a_{j}\right)} \geqslant n
$$

The methods of this paper also provide an easy proof of the following theorem, a weaker version of Szegedy's result. 
THEOREM 2. Let $\left\{a_{i}\right\}_{i=1}^{n}$ and $s$ be as in Theorem 1 but without the condition $s \leqslant 5$. Then for sufficiently large $n$

$$
\max \frac{a_{i}}{\left(a_{i}, a_{1}\right)} \geqslant n
$$

i.e., for fixed s there is a $n_{0}$ such that the conclusion holds for $n \geq n_{0}$.

2. Definitions, notation. Let $a_{1}<a_{2}<\ldots<a_{n}$ be natural integers, $n \geqslant 2$ and put $A=\left\{a_{1}, a_{2}, \ldots, a_{n}\right\}$. Denote the quotient $a_{i} /\left(a_{i}, a_{j}\right)$ by $g_{i j}$. We shall also use $g(a, b)=a /(a, b)$. Let the least common nultiple of the members of $A$ have the decomposition into different primes $p_{1}^{\alpha_{1}} p_{2}^{\alpha_{2}} \ldots p_{s}^{\alpha_{s}}$. Then each $a_{i}$ has the form

$$
a_{i}=p_{1}^{\alpha_{1 i}} p_{2}^{\alpha_{2 i}} \ldots p_{s}^{\alpha_{s i}} \quad \text { with } \quad 0 \leqslant \alpha_{m i} \leqslant \alpha_{m}
$$

and therefore $q_{i j}=p_{1}^{\gamma_{1}} p_{2}^{\gamma_{2}} \ldots p_{s}^{\gamma_{s}}$ with $0 \leqslant \gamma_{m} \leqslant \alpha_{m i}$

A set 1 will be called good respectively bad according as it satisfies (00) or not.

Since in our consideration only the set $\left\{g_{i j}\right\}$ matters we can and shall assume that the largest common divisor of the members in $A$ is 1 . We shall refer to this as Assumption A.

We shall also assume that the primes occurring in (0) are the first primes, i.e., $p_{1}=2, p_{2}=3, p_{3}=5, \ldots$ This is justified by observing that on replacing any $s$ primes by the tirst $s$ primes the corresponding $q_{i j}$ 's will not increase,

A well-known [5] observation is that if $M$ is the least common multiple of the members of $A$ then the set $\left\{g_{i j}\right\},\{i, j\} \subset\{1,2, \ldots, n\}$ is the same as

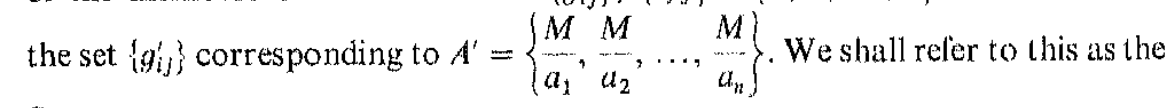
Symmetry property.

\section{Some basic propositions and consequences.}

Propositron 1. Let $A_{n}\left\{p_{i}\right\}$ is: 1 and $\left\{x_{i}\right\}=1$ be as above lf for some mand $i$

$$
p_{m}^{\alpha_{m i}} \geqslant n
$$

then A is good.

Proof. By Assumption A there is some $j$ such that $a_{j}$ is not a multiple of $p_{m}$; then $g_{i j} \geqslant p_{m}^{\alpha_{m i}} \geqslant n$.

Corollary 1. If $A$ is bad then for $m=1,2, \ldots, s$ and $i=1,2, \ldots, n$

$$
0 \leqslant \alpha_{m i}<\frac{\log n}{\log p_{m}}
$$

DFFINITION 1. For given $n$ denote the set of integers of the form

$$
b=p_{1}^{\beta_{1}} p_{2}^{\beta_{2}} \ldots p_{s}^{\beta_{s}} \quad \text { with } \quad 0 \leqslant \beta_{m}<\frac{\log n}{\log p_{m}}
$$

by $\boldsymbol{B}_{s}(n)$. Further denote the number of nembers of $\boldsymbol{B}_{s}(n)$ by $N_{s}(n)$.

OBSERVATION 1

$$
N_{s}(n)=\prod_{m=1}^{n}\left\lceil\left[\begin{array}{c}
\log n \\
\log p_{m}
\end{array}\right\rceil\right.
$$

Corollary 2. If A is bad then

$$
A<B_{s}(n) \quad \text { and } \quad n \leqslant \prod_{m=1}^{s}\left\lceil\frac{\log n}{\log p_{m}}\right\rceil \text {. }
$$

It is convenient sometimes to use

Corollary 3. If $n>N_{s}$ (n) then $A$ is good.

Proposstion 2. There is an integer $n_{1}$ depending only on s such that

$$
n \geqslant n_{1} \quad \text { implies } \quad \frac{N_{s}(n)}{n}<1 \text {. }
$$

Proof. This can be seen by elementary calculus, using

$$
N_{s}(n) \leqslant\left\lceil\frac{\log n}{\log 2}\right\rceil^{s}
$$

DeFinTtron 2. An integer $n^{\prime}$ will be called good if (3) holds for $n^{\prime}=n_{1}$. COROLl.Ary 4. If $|A|=n$ and $n \geqslant n^{\prime}$ and $n^{\prime}$ is good then $A$ is good.

PROPOSTr'on 3. If $B_{s}(n)$ contains two members $b_{1}$ and $b_{2}$ such that one of $g\left(b_{1}, b_{2}\right)$ and $g\left(b_{2}, b_{1}\right)$ is at least $n$ then if $A$ is bad it does not contain both.

Propesition 4. If $\boldsymbol{B}_{s}(n)$ contains $k$ pairwise disjoint pairs $b_{i}, b_{j}$ as $i n$ Proposition 3 then $n>N_{s}(n) \ldots k$ implies $A$ is good.

Proposition 5, let $A$ be $\left\{a_{1}, a_{2}, \ldots, a_{n}\right\}$, and as before $a_{1}<a_{2}<\ldots$ $<a_{n}$. If there is no bad subset of $d$ containing $a_{i}$ then there is no such subset containing $a_{n+i+1}$.

Proof. This follows from the Symmetry property.

Proof of Theorem 2. For any $n \geqslant n_{1}$, where $n_{1}$ is good, the assumption of Corollary 3 is fulfilled; therefore $A$ is good, i.e., $n_{0}$ in Theorem 2 can be taken to be $n_{1}$.

Remark 1. Since " 2 is good for every s" would mean that there are no exceptions to Szegedy's theorem, it is clearly of interest to determine good values as small as we can. We shall do that in Section 4.1. 
4. Proof of Theorem 1. The proof is based on facts from Section 3 and on numerical results obtained by computation. In particular the theorem will follow straight away from Proposition 6 in 4.2 and Proposition 7 in 4.3 .

4.1. An algorithm. It is not too difficult to decide for a fixed s whether a set $N_{s}$ of $N_{s}$ numbers contains a bad set of size $n_{s}$ or less if $N_{s}$ and $n_{s}$ are not too large. We could formulate and program on a computer such an algorithm, based essentially on trial and error and have the answer in reasonable time if $N_{s} \leqslant 100000$ with $n_{s} \leqslant 150$, while for $N_{s} \leqslant 15000$ even with $n_{s} \leqslant 7500$. These bounds are sufficient for our purposes and are not sharp. In this way the first step in proving Theorem 1 is made.

\subsection{A procedure for ohtaining smallest good integers.}

\section{Procedure 1.}

Suppose $n^{(1)}$ is good; put $N_{s}\left(n^{(1)}\right)=l_{1}$ and $n^{(2)}=l_{1}+1$. Then $N_{s}\left(n^{(2)}\right) \leqslant N_{s}\left(n^{(1)}\right)<n^{(2)}$, and $n^{(2)}$ is a good value smaller than $n^{(1)}$, provided $l_{1}+1<n_{1} ;$ notice $l_{1}+1 \leqslant n_{1}$ holds always.

Repeating the above procedure one defines a sequence

$$
n^{(1)}>n^{(2)}>\ldots>n^{(k)}=n^{(k+1)}
$$

and the value $n^{(k)}$ cannot be improved in the same way.

Defrnition 3. The smallest good value which may be obtained by Procedure 1 will be denoted by $n^{(0)}$ and $n^{0}(s)$ emphasizing $s$.

Example 1. Put $s=2$, i.e., $p_{1}=2, p_{2}=3$, then 64 is a good value, since

$$
\left.24=6 \cdot 4=\left\lceil\frac{\log 64}{\log 2}\right\rceil \frac{\log 64}{\log 3}\right\rceil<64
$$

Put

$l_{1}=24, n^{(1)}=64$, then $n^{(2)}=25$.

$l_{2}=\left[\begin{array}{c}\log 25 \\ \log 2\end{array}\right]\left[\begin{array}{c}\log 25 \\ \log 3\end{array}\right]=5 \cdot 3=15$, then $n^{(3)}=16$.

$\left.l_{3}=\left[\frac{\log 16}{\log 2}\right] \frac{\log 16}{\log 3}\right]=4 \cdot 3=12$, then $n^{(4)}=13$.

$\left.l_{4}=\left\lceil\frac{\log 13}{\log 2}\right\rceil \frac{\log 13}{\log 3}\right\rceil=4 \cdot 3=12$, therefore $n^{(0)}=13$.

Proposimion 6. $n^{0}(2)=13, n^{0}(3)=160, n^{0}(4)=1540, n^{0}(5)=33600$.

Proof. The above values can be obtained by computation using Procedure 1.

Remark 2. Proposition 6 shows that smallest good values are no good enough to prove that for fixed $s$ Szegedy's theorem is true with no exceptions.
Definition 4. An integer $n^{\prime \prime}$ is bad if for every bad it follows that $n \leqslant n^{\prime \prime}$. Notice that here (3) is not used.

COROIARY 5. $n_{1}=n^{(0)}-1$ is had.

Remark 3. "1 is bad" would mean that there are no exceptions, so it is of interest to find small bad values.

\subsection{A procedure for obtaining small bad values.}

\section{Procedure 2.}

Consider $\boldsymbol{B}_{s}\left(n_{s}^{(1))} \ldots-1\right)$; determine as many as possible pairs $b_{i}, b_{j}$ as in Proposition 3. Suppose there are $k_{1}$ such pairs. Put $\pi_{2}=N\left(\pi_{1}\right)-k_{1}$. If $k_{1}>0$ then this value is bad and certainly smaller than $\pi_{1}$. Repeating this procedure one defines a sequence $n_{1}>m_{2}>\ldots>\pi_{k}^{n}=n_{k}+1$ where $\pi_{k}$ cannot be improved by this procedure.

DEFINITION 5 . Write $\tilde{n}$, and emphasizing $s, \vec{n}(s)$, for the smallest bad value which can be obtained in this way.

EXAMPLE 2 . Let $s=2$. Then $\tilde{n}_{1}(2)=n_{2}^{(0)}-1=12$.

$$
B_{2}(12)=\left\{\begin{array}{rrrr}
1 & 2 & 4 & 8 \\
3 & 6 & 12 & 24 \\
9 & 18 & 36 & 72
\end{array}\right\}
$$

Observe that the 4 pairs $\{1,12\},\{2,24\},\{3,36\},\{6,72\}$ are as required. Therefore $\pi_{2}=12-4=8$; then consider

$$
B_{2}(8)=\left\{\begin{array}{rrrr}
1 & 2 & 4 & 8 \\
3 & 6 & 12 & 24
\end{array}\right\}
$$

containing the pairs $\{3,24\},\{1,8\}, \vec{n}_{3}=8-2=6$,

$$
B_{2}(6)=\left\{\begin{array}{rrr}
1 & 2 & 4 \\
3 & 6 & 12
\end{array}\right\}
$$

with pairs $\{2,12\},\{1,6\}, \pi_{4}=6-2=4, B_{2}(4)=\{1,2,3,4\}$ containing $\{1,4\}, \bar{n}_{5}=4-1=3, B_{2}(3)=\{1,2,3\}$ containing $\{1,3\}, \bar{n}_{6}=2, B_{2}(2)$ $=\{1,2\}, \bar{n}_{2}=1$ so $n=1$.

PropostrION 7. $n(2)=1, \vec{n}(3)=44, \bar{n}(4)=759, \vec{n}(5)=7350$.

Proof. The above values are obtained by Procedure 2.

Final remark. As already mentioned the numerical results in Sections 4 are not sharp. To improve Theorem 1, i.e., to prove its validity for values of $s$ larger than 5 , one should apply more powerful tools than those used in Section 4 
Another way to improve our results would be to replace the inequality in Corollary 2 by a stronger incequality, namely

$$
n \leqslant c_{s} \prod_{m=1}^{s}\left[\begin{array}{c}
\log n \\
\log p_{m}
\end{array}\right\rceil
$$

where $c_{s}$ depends only on s and decreases rapidly with it. Some heuristic evidence, based on geometric considerations and on a relinement of the Symmetry property, indicates the existence of such a constant; this could lead to prove Szegedy's theorem with no exeeptions.

Finally, this would also follow from the following conjecture in the spirit of [3].

Consferurt: (Schönheim). If $a_{1}<a_{2} \ldots \ldots a_{n}, n \geq 2$ are natural integers and $a_{n} / a_{1}<n$ then

$$
\left|\left\{\begin{array}{c}
a_{i} \\
\left.\ldots a_{i}, a_{j}\right)
\end{array}\right\}\right| \geqslant n
$$

\section{References}

[1] R. D. Boyle, On a problem of R. L. Groham, Astn Arith. 34 (14)68), 197. 1613177

[2] R. L. Graham, Unsolved problem 5740, Amer. Mith. Montliy 77 (197(0), p. 775.

[3] J. Marica and J. Schonheim, Differences of sets and " prohl'm of circhum, Cinatd. Math. Bull. $12(1969)$, pp. 6356.37

[4] M. Szegedy, The wolution of Graham's ( $G\left({ }^{1} D\right.$ problem (to appear).

[5] W. Y. Vélez, Some remarks on a number theoretic problent of (irahtom, Acta. Arth. 32 (1977) pp. $233 \cdot 238$.

[6] G. Weinstein, On a conjecture of (irahum concerning yreatest common divisors, Proc. Amer Math. Soc. 63 (1977), pp. 33-38

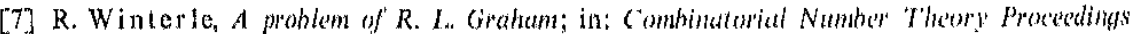
Louisiana Conference on Combinatories, Baton Rouge 1070. p1) 357.361 .

SCHOOH, OH MATHIMATHAL, SCIENTS

IINIYLESTITY

Tel-Aviv, tartiel 